\title{
Additional measures for bedside endoscope cleaning to prevent contaminated splash during COVID-19 pandemic
}

We read the recent European Society of Gastrointestinal Endoscopy (ESGE) and European Society of Gastroenterology Nurses and Associates (ESGENA) Position Statement on gastrointestinal endoscopy and the COVID-19 pandemic with great interest. The Statement provides guidance to protect against COVID-19 for both patients and endoscopy personnel. We are concerned, however, that the general comment number 3 [1], which states, "Reprocessing of flexible endoscopes and endoscopic accessories should be performed according to published guidelines," is not appropriate in the setting of COVID-19 contaminated endoscopes. The ESGE and ESGENA guidelines in 2018 [2] recommend a precleaning step, which, in the context of COVID-19 contamination, may cause endoscopy personnel to be unnecessarily splashed.

Therefore, during precleaning at the bedside [2], we recommend first applying virucide to the endoscope control section and insertion tube by using a gauze dampened in $70 \%$ alcohol. Sec- ond, to remove debris and to prevent biofilm formation in the endoscope channel, we recommend using enzymatic detergent solution [3]. The use of enzymatic detergent is preferred because it reduces the amount of organic residue compared with the use of nonenzymatic detergent [4]. Notably, we strongly recommend against suction or blowing air, or flushing water into any of the channels as these actions may cause splashing or even aerosolization ( $\mathbf{F i g . 1 )}$. Thus, we recommend only suctioning the detergent through the working channel [4]. The valves should not be replaced before finishing this process because significant splashing may occur during the valve exchange. Third, we recommend immersing the endoscope in a bin containing $0.2 \%-0.35 \%$ paracetic acid, glutaraldehyde, or ortho-phthalaldehyde solution for the duration recommended by the manufacturer ( $>$ Fig. 1 ). These solutions are virucidal. Ultimately, the endoscope can be transferred to the reprocessing room and undergo the standard reprocessing order, starting from leak testing, repeat manual cleaning (this time with flushing, rinsing etc.), and standard high-level disinfection [2].

The person who performs the bedside precleaning process should wear an enhanced set of personal protective equipment (PPE) and, in order to make best use of available PPE, that person can be the assistant who is already wearing appropriate PPE. In such a situation, the person in the reprocessing room may wear standard PPE (> Fig.2) [1]. When the additional safety measures can only be performed in the reprocessing room, the person responsible must wear an enhanced set of PPE.

To win the war against COVID-19, we must prevent all healthcare personnel from becoming infected and further spreading the virus to their communities. As the standard protocol may cause splashing or even aerosolization, steps must be taken to mitigate the transmission risk. While waiting for the study to prove the benefit of these additional

\section{Endoscope reprocessing during COVID-19 outbrak}

Endoscopy in PUI/or confirmed COVID-19 case Additional pre-cleaning process
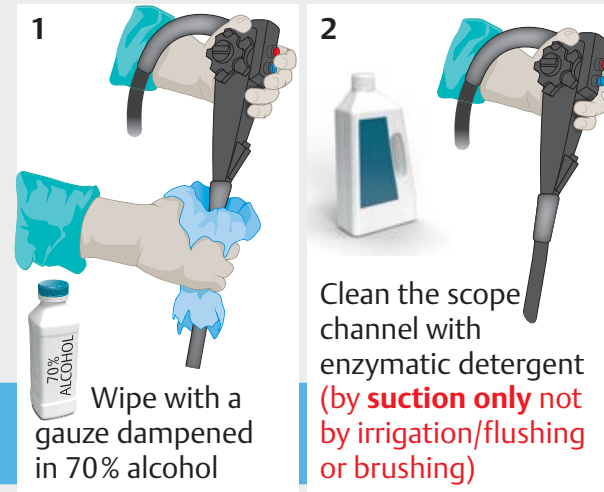

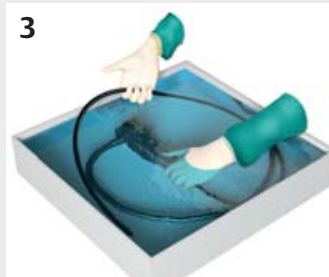

Immerse in 0.2-0.35\% paracetic acid or glutaraldehyde or OPA (duration as per manufacturer recommendation)

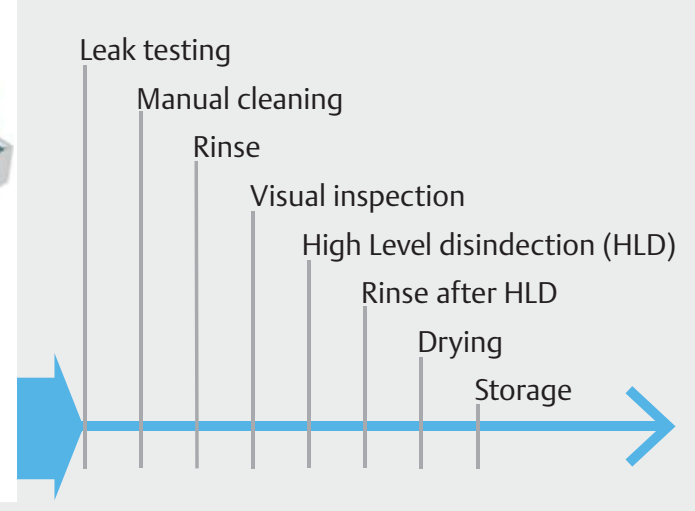

- Fig. 1 Endoscope reprocessing with additional measures for bedside endoscope cleaning during the COVID-19 outbreak. PUI, person under investigation; OPA, ortho-phthalaldehyde. 


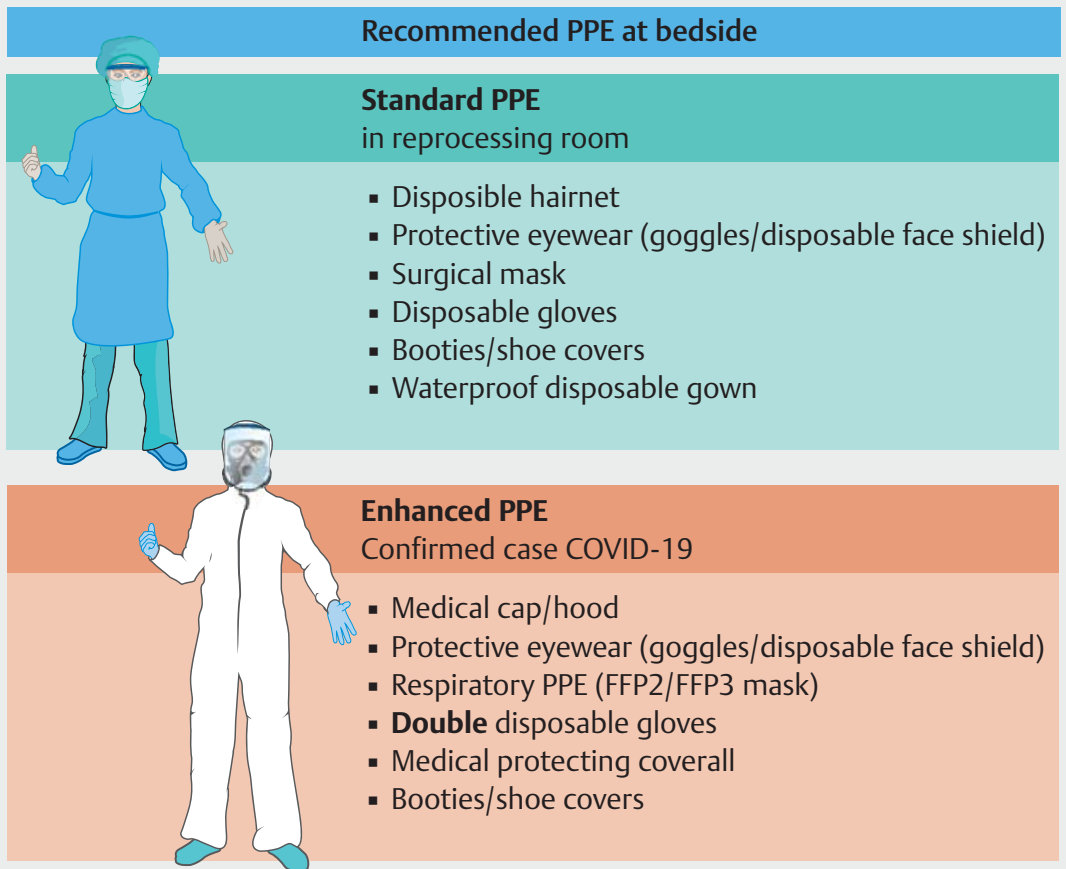

Position statements on COVID-19: APSDE, ASGE and CDC 2020

- Fig. 2 Personal protective equipment (PPE) for the person who performs the bedside precleaning process and the person in the reprocessing room, according to 2020 position statements on COVID-19 from Asian Pacific Society of Digestive Endoscopy, American Society for Gastrointestinal Endoscopy, and Centers for Disease Control and Prevention.

steps, we believe that "to do more" is essential at present.

\section{Competing interests}

The authors declare that they have no conflicts of interest.

The authors

Rungsun Rerknimitr ${ }^{1,2}$, Roy Soetikno ${ }^{3}$, Thawee Ratanachu-Ek ${ }^{4}$, Kasenee Tiankanon ${ }^{1}$, 2, Pradermchai Kongkam ${ }^{1,2}$, Wiriyaporn Ridtitid ${ }^{1,2}$

\section{Corresponding author}

\section{Rungsun Rerknimitr, MD}

Division of Gastroenterology, Department of Medicine, Faculty of Medicine,

Chulalongkorn University, 2nd floor, Bhumisiri Bldg, King Chulalongkorn Memorial Hospital, 1873 Rama IV Rd, Bangkok 10330, Thailand

Fax: +66-2-2527839

ERCP@live.com

\section{References}

[1] Gralnek IM, Hassan C, Beilenhoff U et al. ESGE and ESGENA Position Statement on gastrointestinal endoscopy and the COVID19 pandemic. Endoscopy 2020: doi:10.1055/a-1155-6229

[2] Beilenhoff U, Biering H, Blum R et al. Reprocessing of flexible endoscopes and endoscopic accessories used in gastrointestinal endoscopy: Position Statement of the European Society of Gastrointestinal Endoscopy (ESGE) and European Society of Gastroenterology Nurses and Associates (ESGENA) update 2018. Endoscopy 2018; 50: 12051234

[3] Thaker AM, Kim S, Sedarat A et al. Inspection of endoscope instrument channels after reprocessing using a prototype borescope. Gastrointest Endosc 2018; 88: 612-619

[4] Gonzalez JA, Vanzieleghem T, Dumazy A et al. On-site comparison of an enzymatic detergent and a non-enzymatic detergent-disinfectant for routine manual cleaning of flexible endoscopes. Endosc Int Open 2019; 7: E412-E420

2 Center of Excellence for Innovation and Endoscopy in Gastrointestinal Oncology, Chulalongkorn University, Bangkok, Thailand

3 Division of Gastroenterology and Hepatology, San Francisco Veterans Affairs Medical Center, San Francisco, California, USA

4 Department of Surgery, Rajavithi Hospital, Bangkok, Thailand 